\title{
Redes académicas en la investigación en Comunicación en Chile: análisis de co-autorías en el trabajo científico
}

\author{
Daniela Lazcano-Peña*, Danilo Reyes-Lillo** \\ *Pontificia Universidad Católica de Valparaíso, Chile \\ Correo-e: daniela.lazcano@pucv.cl | ORCID iD: https://orcid.org/0000-0002-3251-7747 \\ **Universidad de Playa Ancha, Chile \\ Correo-e: danilo.reyes@upla.cl | ORCID iD: https://orcid.org/0000-0002-0141-8324
}

Recibido: 19-11-2018; 2a versión: 08-04-2019; Aceptado: 14-04 -2019.

Cómo citar este artículo/Citation: Lazcano-Peña, D.; Reyes-Lillo, D. (2020). Redes académicas en la investigación en Comunicación en Chile: análisis de co-autorías en el trabajo científico. Revista Española de Documentación Científica, 43 (1), e259. https://doi.org/10.3989/redc.2020.1.1626

Resumen: Se presenta una mirada al campo de la investigación en Comunicación en Chile desde el análisis de sus redes de colaboración académica, operacionalizado a través de la caracterización de las co-autorías de 57 proyectos de investigación financiados con fondos públicos, entre los años 2010 y 2016, y una muestra de 417 artículos académicos publicados en revistas científicas nacionales e internacionales del área entre 2010 y 2018 . Estos resultados se complementan, desde una perspectiva cualitativa, con entrevistas sobre la valoración del trabajo en red, aplicadas a una muestra de investigadores e investigadoras vinculadas al área en Chile. Como principales resultados se observa una predisposición positiva al trabajo en red, pero dificultado por las actuales condiciones de exigencia a la productividad científica, en que se desarrolla el trabajo académico hoy.

Palabras clave: investigación en Comunicación; campo académico; co-autorías; redes de colaboración; colaboración científica.

\section{Academic networks in Communication research in Chile: analysis of co-authorships in scientific work}

\begin{abstract}
In this article, we present a view at the field of Communication research in Chile, from the analysis of its networks of academic collaboration, operating through the characterization of co-authorship of 57 research projects, financed with public funds between 2010 and 2016, along with a sample of 417 academic articles published in national and international scientific journals of the area between 2010 and 2018. These results are complemented, from a qualitative perspective, with interviews about the assessment of networking, one sample of researchers linked to this area in our country. The results show a positive predisposition for networking which, nevertheless, is partially hindered by the current demand conditions for scientific productivity in which academic work is developed nowadays.
\end{abstract}

Keywords: research in Communication; academic field; co-authorships; collaboration networks; scientific collaboration.

Copyright: ( 2020 CSIC. Este es un artículo de acceso abierto distribuido bajo los términos de la licencia de uso y distribución Creative Commons Reconocimiento 4.0 Internacional (CC BY 4.0). 


\section{INTRODUCCIÓN}

La ciencia en tanto práctica social (Aguado-López y otros, 2018), y con ella la investigación científica, se constituye notoriamente como consecuencia de un contexto de producción determinado. Considerando el entorno en el que se inserta, viene a ser un resultado empapado de las características, transformaciones y renovaciones propias de la época y su ambiente epistemológico inmediato. Por ello, no es sino considerando este tipo de perspectivas más o menos históricas que puede entenderse mejor la elaboración y publicación de artículos, la conformación de comunidades y los hábitos científicos de éstas.

En la actualidad, la ciencia, envuelta en una sociedad hiperconectada, compleja, con vínculos interdisciplinares y un abanico de tecnologías en constante crecimiento, logra saltar distancias y amplía la posibilidad de co-construir conocimiento. Desde el siglo XX una multiplicidad de factores ha configurado el que es, probablemente, uno de los rasgos más distintivos de la producción científica en el último tiempo: el trabajo colaborativo, en red y, como consecuencia, la co-autoría.

La colaboración entre investigadores se constituye como un rasgo definitorio de la ciencia actual (González y Gómez, 2014), expresado en vínculos formales o informales entre investigadores (Aguado-López y otros, 2018). Es un fenómeno que dado su contexto parece estar en auge y, más allá de ser una posibilidad, asoma como una necesidad: el interés por ser parte de comunidades científicas, la urgencia por mantener vigencia y un ritmo apropiado de publicación, complementar habilidades o internacionalizar el alcance del estudio a través de la integración de prácticas de investigación locales hacia prácticas globales son algunas de las razones por las que este trabajo en red ha tomado tanta fuerza (Jung y Ruiz-León, 2018). La colaboración científica, entonces, y si bien no es una característica nueva en el desarrollo de la ciencia (Finholt y Olson, 1997), es sin duda parte esencial -hasta una necesidad (Edwards y otros, 2011)- y del momento que vive hoy la ciencia y su proceso productivo de conocimiento.

De acuerdo a la revisión que proponen González Alcaide y Gómez Ferri (2014), el auge del trabajo colaborativo se ha incrementado desde mediados del siglo $\mathrm{XX}$, impulsado en especial por el desarrollo de las tecnologías de la información, que han facilitado el contacto y configuración de grupos de investigadores de diferentes instituciones y países, materializado en experiencias de investigación a gran escala como los estudios de big science y bases de datos colaborativas que reúnen a miles de científicos alrededor del mundo (López-Borrull y Canals 2013), y fomentado incluso por su incorporación en políticas públicas de fomento de la investigación y desarrollo científico (González Alcaide y Gómez Ferri, 2014) en diferentes partes del mundo. En el caso de Chile, por ejemplo, desde inicios del siglo XXI el principal ente público de apoyo a la investigación científica -Conicyt- considera como parte de sus programas de fomento la Cooperación Internacional -PCIpara "incentivar la vinculación y fortalecimiento de redes mundiales con el propósito de incorporar a la comunidad científica nacional el conocimiento de vanguardia" (Conicyt, 2019) y, desde 2009, cuenta con el Programa de Investigación Asociativa -PIA- para la promoción de centros de investigación de excelencia (Conicyt, 2019).

El auge de la colaboración científica ha derivado también en su observación como objeto de estudio con trabajos pioneros en la década de los 60 (Price, 1963; Price y Beaver, 1966). Desde una mirada teórica, las primeras aproximaciones a esta línea de estudio se han encontrado en la historia o la sociología de la ciencia y, desde una tendencia más contemporánea, en la bibliometría y el análisis de redes sociales (González Alcaide y Gómez Ferri, 2014) como propuestas teóricas y metodológicas. Dentro de la amplia documentación bibliográfica desarrollada, es posible observar análisis, por ejemplo, que se introducen en las diferencias que pudiesen existir entre diferentes áreas disciplinarias. Desde este enfoque, y aunque se reconoce la colaboración científica como un elemento predominante en las ciencias más "duras" o físicas, se evidencia un claro desplazamiento a otras áreas más vinculadas a las Ciencias Sociales (Finholt y Olson, 1997) Artes y Humanidades (Gómez Ferri y González Alcaide, 2018). En otras palabras, estamos ante una forma de generación y desarrollo de la Ciencia, transversal a todos los campos de conocimiento.

Dentro del estudio sobre las redes de colaboración científica, en esta investigación nos centramos en una área disciplinaria específica: las Ciencias de la Información y la Comunicación. Si bien son escasas las aproximaciones centradas en este campo del conocimiento en particular, sí podemos encontrar algunos trabajos que, en el marco de caracterizaciones mayores sobre el estado de la comunidad científica de la Comunicación, aportan datos específicos sobre la evolución de las redes de colaboración en esta área. Así, y considerando el caso español por ejemplo, una observación de coautorías presentes en 1.182 artículos publicados entre 2007 y 2011 en las más valoradas revistas científicas de Comunicación en España, evidencia 
una irreversible disminución de la investigación del trabajo colectivo, motivado por los estándares internacionales y las exigencias de publicación (Escribà y Cortiñas, 2013). Es decir, se asume -aunque veremos más adelante que el argumento puede ser observado críticamente (González Alcaide y Gómez Ferri 2014)- que la colaboración científica, operacionalizada en este caso en las co-autorías, podría aportar una mayor calidad al trabajo científico en esta área (Martínez-Nicolás y Saperas 2011, 2016)

Siguiendo el recorrido de lo macro a lo micro -es decir, desde el estudio de las redes de colaboración en el desarrollo de la ciencia en general a áreas disciplinarias particulares- en este artículo analizamos la generación de conocimiento en el campo de la Comunicación en Chile, tenido en cuenta que es un área creciente en distintas dimensiones: en cuanto al número de programas de formación especializada (27 universidades con pregrado, 32 con magíster y 3 con planes doctorales), en la disposición de espacios de difusión académica (revistas especializadas y un congreso nacional organizado desde 2014 por la Asociación Chilena de Investigadores en Comunicación - InCom Chile) y también en el desarrollo de un ejercicio auto-reflexivo a través de la metainvestigación del campo, cuyo interés y producción académica ha vivido un creciente auge en los últimos años (Dittus, 2008; Del Valle, 2004; Lazcano-Peña, 2007; Caldevilla y Del Valle 2011; Lazcano-Peña y Perry, 2016; Lagos, 2018).

En esta línea de meta-análisis de la propia área de estudio, se inserta también este trabajo, enmarcado en el proyecto "Investigación en Comunicación en Chile: estado del arte y análisis de las características y proyecciones de la comunidad científica nacional" [COD Fondecyt 11160842], cuyo objetivo es caracterizar el campo académico de la Comunicación en Chile, considerando sus dimensiones científica - prácticas de producción de conocimiento de los investigadores e investigadoras - y educativa - reproducción de conocimiento a través de la docencia universitaria.

Si bien el proyecto marco considera la comprensión del campo desde múltiples variables -trayectorias académicas, institucionales y disciplinarias de los/as investigadores e investigadoras; temáticas, objetos y problemas de investigación predominantes, enfoques metodológicos, entre otras variables- en este artículo en específico buscamos analizar las relaciones y los vínculos de colaboración académica que se observan en la generación de conocimiento científico en el campo de la Comunicación en Chile, combinando enfoques cuantitativos y cualitativos. Así, los resultados son consecuencia de la aplicación de técnicas provenientes de la cienciometría y el estudio de redes, comple- mentadas con el análisis de una serie de entrevistas aplicadas a una muestra de investigadores e investigadoras vinculados al campo. De este modo, se logra sistematizar tanto las redes, como las valoraciones y significaciones con las que la propia comunidad académica comprende y explica sus lógicas colaborativas en la producción de conocimiento científico.

\section{ANTECEDENTES Y OBJETIVOS}

El interés de investigar la investigación y sus investigadores e investigadoras, es decir, el enfoque de meta-investigación de este estudio, se sustenta en una motivación inicial por aproximarse al debate epistemológico sobre la Comunicación. En ese sentido, entendemos que la discusión sobre la configuración disciplinaria de la Comunicación puede ser abordada desde perspectivas históricas, teóricas o epistemológicas, por ejemplo. En nuestro caso nos aproximamos a esta discusión desde una de las aristas materiales u operacionales de su consolidación institucional: su comunidad científica.

Comprendemos el concepto de comunidad científica desde la noción original de Kuhn (1975), para quien la ciencia se materializa en una comunidad "formada por practicantes de una especialidad científica". En ese sentido, entendemos que centrar la mirada en el quehacer de los propios investigadores e investigadoras, nos permitirá conocer y caracterizar como se materializa, en la práctica, el desarrollo de la disciplina.

Esta perspectiva se sistematiza, además, con la propuesta de Vassallo de Lopes (2001), quien -y en base a la noción de campo de Bourdieu- conceptualiza el campo académico de la Comunicación, como "un conjunto de instituciones de educación superior destinadas al estudio y a la enseñanza de la comunicación, donde se produce la teoría, la investigación y la formación universitaria de los profesionales de la comunicación", noción que divide, a la vez, en subcampos científico (prácticas de producción de conocimiento); educativo (prácticas de reproducción de ese conocimiento); profesional (prácticas de aplicación).

Tomando ambas perspectivas - la idea de comunidad científica de Kuhn (1975) y la comprensión de la organización de campo académico de Vassallo de Lopes (2001), entendemos que el análisis de aquellos elementos en que se expresa el quehacer de la comunidad en el campo, entregará información sobre la forma en que se articula y desarrolla la generación de conocimiento. En esta observación concreta, este interés lo enfocamos en la articulación del trabajo de los investigadores, para aproximarnos a la configuración de un mapa de 
las redes de intelectuales, es decir, a "la existencia de contactos profesionales durante un período de años entre un conjunto de personas que se reconocen como pares y que de manera consciente utilizan esos contactos para promover algún tipo de actividad profesional" (Devés 2004).

Si bien la comprensión de redes de intelectuales implicaría, siguiendo a Devés (2004), una consistencia temporal, sus primeros pasos de configuración serán siempre un espacio de trabajo conjunto desarrollado como actividad de colaboración científica. Sobre esta lógica de producción de conocimiento, González y Gómez (2014) apuntan que "de entrada, una perspectiva histórica revela una gran variedad de formas y tipos de colaboración que se van transformando en relación con su contexto, y una diversidad de niveles de análisis y de actores involucrados, que van desde individuos a países, pasando por grupos y organizaciones. Debido a la complejidad del fenómeno, con el fin de estudiarla, se puede abordar la colaboración científica como un espacio de prácticas e interacciones sociales extendido en el tiempo" (p. 4).

Como ya se ha indicado, y si bien la literatura encuentra antecedentes sobre los estudios de redes de de colaboración en los años 60 , ya entrando en los 70 era notoria la publicación de trabajos con multiautoría. Las redes de colaboración informales entre autores fueron constituyendo los llamados "colegios invisibles" y la reunión de investigadores con figuras centrales dotadas de cierto prestigio productivo se hicieron cada vez más comunes. Así, con el auge de la colaboración en ciencias, dos principales cuestionamientos necesitaron ser respondidos: el primero, en relación a la delimitación de la vinculación entre investigadores como fenómeno y el segundo sobre la metodología para estudiarla (González y Gómez, 2014). El análisis bibliométrico resolvió las dudas, pero dirigió la colaboración hacia un área específica: la co-autoría. Esto, pues si bien se entiende que la co-autoría es una de muchas formas de colaboración, sintoniza bien con una lógica cuantitativa de medición, funcional al entorno científico-político que rodea la producción de conocimiento, donde la adjudicación de proyectos y la publicación de artículos aumentan su valía como indicadores del trabajo académico.

Entonces, como motivantes de la asociación, las razones propias de la producción de conocimiento -pertenecer a un grupo investigativo, complementar habilidades y dominios disciplinares, adquirir experiencias y generar un aporte local significativo, por mencionar algunas - fueron subordinadas a la necesidad de hacer frente a las políticas de evaluación de la producción de conocimiento. La sobrevivencia viene a ser el objetivo principal. De ahí surge el paradigma publicar o perecer que se transforma en asociarse o perecer (Aguado-López y otros, 2018).

En el caso particular de las ciencias sociales la situación es compleja por sí misma. Los estudios en este campo "no buscan explicar, ni emitir verdades, sino que se centran en discutir cosas tan importantes para el ser humano como su identidad, el significado de su mundo, la lengua que nombra todas las cosas, su memoria" (Carrasco, 2013), de modo que es difícil establecer generalizaciones o consensos, dificultando la asociación más que en otras disciplinas. Por lo mismo, la coautoría y la colaboración, en general, entre investigadores debiera tener especial cuidado y necesariamente poner por delante el contenido y la contribución local que se puede hacer con la producción de conocimiento antes de la estadística. Sin embargo, considerando las exigencias del sistema científico y el camino estandarizado de aportación, la presión del medio termina por someter a los investigadores a la institucionalidad. Lo que sin duda es válido si, como hemos mencionado, se intenta sobrevivir académicamente.

Entonces las motivaciones de los investigadores por publicar y asociarse se sustentan en dos aspectos principales: la productividad y la calidad. Ambas dimensiones permeadas absolutamente por el sistema político, histórico, social, científico en el que se desenvuelven, de modo tal que la productividad no es otra cosa que un afán por publicar mucho en poco tiempo y la calidad se asegura si tales publicaciones superan los estándares de revistas mejores calificadas (Aguado-López y otros, 2018).

Para concluir, cabe destacar y resumir que el panorama de colaboración científica parece no responder por completo al deseo de generar conocimiento significativo con impactos locales, sino que, por el contrario, tiende a ser motivado por razones externas y propias del medio: complementar habilidades, acceder a mejores equipos, alcances y condiciones generales de investigación, obtener reconocimiento y visibilidad, incrementar la productividad y enfrentar la institucionalidad son los motivantes principales de los investigadores para asociarse (Aguado-López y otros, 2018; GómezFerri y González-Alcaide, 2018)

Sin embargo, como se plantea en este estudio, la ciencia es una práctica social y sus implicaciones contextuales no pueden ignorarse. La actitud de los investigadores, o dicho de otro modo, el hábito de las comunidades científicas tendiente a la productividad y a la satisfacción de las exigencias de la institucionalidad, son consecuencia del ambiente en el que se desenvuelven y parte de un rasgo 
característico de la ciencia moderna. No obstante, cabe preguntarse si la generación de conocimiento y su aporte es el fin que se persigue o sólo un paso previo y necesario para otros objetivos. De todos modos, lo cierto es que la asociación de sujetos tiene un componente claro de supervivencia: dos o más autores son mejores que uno si se quiere tener presencia y visibilidad en el campo.

Desde la óptica del análisis bibliométrico, el análisis de co-autoría y la colaboración científica toman como fuente de datos la firma de artículos científicos, considerando para cada artículo el número de autores, su posición en la firma, su afiliación institucional y su país, dependiendo del objetivo del estudio (Todeschini y Bacci, 2016).

Si bien la medición de las co-autorías ha tenido un gran desarrollo a lo largo de los últimos años, hasta llegar a convertirse en predominante en la actualidad, sabemos que existe un profundo debate sobre la relación entre la firma de un paper como indicador válido de colaboración científica (Katz y Martin 1997), en especial por el incremento de malas praxis como conflictos de autorías: autorías inmerecidas, honorarias o fantasmas (González Alcaide y Gómez Ferri, 2014). Asumiendo estas observaciones como llamados de atención crítica, operacionalmente en este trabajo consideraremos, desde la cienciometría, que los datos que otorga la co-autoría de un paper pueden ser interpretados como una evidencia documentada de un proceso de investigación colaborativa (Katz y Martin, 1997; Sonnenwald, 2007). Estas colaboraciones pueden formar una "red de co-autoría", donde los nodos o vértices de la red representan a los actores involucrados en la productividad del paper y las líneas o vínculos entre los nodos representan las relaciones en la producción de artículos, que se realiza escribiendo al menos un paper juntos (Figura 1). En términos generales, la estructura de estas redes de co-autoría pueden caracterizar las comunidades académicas (Newman, 2004).
Los estudios cuantitativos de redes de co-autoría y colaboración científica se llevan a cabo utilizando técnicas y métricas propias del análisis de redes sociales (Wasserman y Faust, 1994), tanto para el estudio a nivel de redes como a nivel de nodos particulares, según el aspecto o la propiedad que se evalúe.

Por una parte, las métricas asociadas a nodos corresponden a aquellos indicadores que describen una propiedad particular de cada uno de los nodos de forma independiente $y$, por consecuencia, se obtiene un valor determinado para cada uno de los nodos de la red. En este tipo de métricas encontramos, por ejemplo, indicadores como el grado nodal, que en redes simétricas o no dirigidas, representa el número de nodos adyacentes que presenta determinado nodo. Asimismo, es posible identificar indicadores de centralidad, de propiedades espectrales o de agrupamiento para cada nodo de la red (Casas-Roma y Pérez-Solà, 2016); o métricas asociadas o aplicadas a nivel de red, que permiten caracterizar y obtener un valor o un conjunto de valores correspondiente a toda la red (Casas-Roma y Pérez-Solà, 2016). Entre las principales métricas de red, podemos identificar el diámetro (que se define como la mayor de las distancias mínimas entre los nodos de la red) y la densidad (cantidad de vínculos existentes en una red, en relación a la cantidad de vínculos posibles), lo que nos dará pistas sobre las características de colaboración -o no- de la red.

Tomando ambas miradas conceptuales -el interés por la meta-investigación en Comunicación y el análisis de redes- el objetivo de este trabajo es, por una parte, (i) caracterizar las redes de colaboración académica desarrolladas por los y las investigadores del campo de la Comunicación en Chile, y (ii) identificar las valoraciones y significaciones con que los y las investigadores comprenden y explican su trabajo colaborativo.

Figura 1. Ilustración de una red de co-autoría
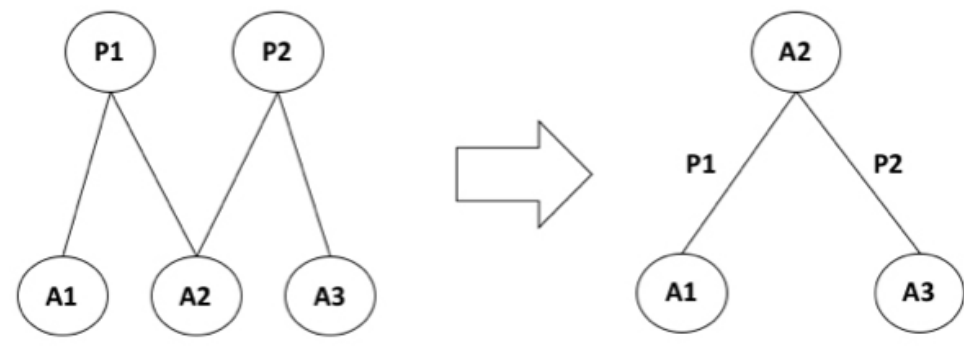

$$
\begin{array}{ll}
\text { A1 }=\text { Autor 1 } & \text { P1 }=\text { Paper 1 } \\
\text { A2 }=\text { Autor 2 } & \text { P2 }=\text { Paper 2 } \\
\text { A3 }=\text { Autor 3 } &
\end{array}
$$




\section{DISEÑO METOdOLÓgICO (MÉTODOS, MATERIALES Y FUENTES)}

Sobre el corpus de investigación de la fase cuantitativa, correspondiente al primero de nuestros objetivos, se hace necesaria una aclaración inicial, pues si bien el estudio de co-autoría se centra principalmente en la firma de papers, en este caso nos enfocamos al estudio de dos fuentes de información. Por una parte, y siguiendo la tendencia de esta línea de investigación, analizamos papers o artículos científicos publicados en una muestra de revistas especializadas en ciencias de la información y la comunicación, nacionales (chilenas) e internacionales. En el caso de las revistas chilenas, acotamos la muestra a artículos publicados entre 2010 y 2018, en cinco revistas de la especialidad. Si bien en Chile existen otras publicaciones vinculadas a la comunicación o áreas afines, la elección de estas revistas se basó en su sostenibilidad en el tiempo y el acceso abierto en versión digital a la totalidad de las ediciones consideradas en el período analizado, lo que garantizó la factibilidad de acceso al material de conformación del corpus de estudio (anexo 1). Del total de textos publicados en las ediciones revisadas, se consideró como parte de la investigación aquellos que contaran con, al menos, un autor de afiliación a alguna universidad chilena, alcanzándose un corpus de 202 textos. En el caso de las revistas internacionales, en tanto, el estudio se acotó a aquellos artículos que contaran con al menos un autor de afiliación a alguna universidad chilena, publicados en revistas que aparecieran en la base de datos de Web of Science (WOS), con los criterios de búsqueda 'comunicación', 'periodismo', 'medios' e 'información', y sus equivalentes en inglés, entre los años 2010 y 2018 . En este caso, el corpus alcanzó a 215 textos (anexo 1).

Sumado al análisis de papers, y ampliando el enfoque de los estudios de cienciometría, incluimos el estudio de proyectos de investigación, el que entendemos y valoramos como el paso previo -generación de conocimiento- a la publicación de artículos -difusión de conocimiento. Con esta intención, trabajamos con una base de datos de los proyectos ejecutados entre los años 2010 y 2016, con apoyo de la Comisión Nacional de Ciencia y Tecnología -Conicyt- principal organismo público dedicado al fomento del desarrollo científico y tecnológico en Chile. En específico, sistematizamos los datos de dos de sus líneas de fondos públicos concursables: proyectos financiados en los concursos 'Fondecyt Regular' y 'Estudios sobre Pluralismo en el Sistema Informativo Nacional'. No se consideraron otras fuentes de financiamiento, públicas o privadas, por la falta de acceso a los datos que hubieran sido necesarios para la revisión íntegra de todas las investigaciones vinculadas al área que se pudieran haber generado por otras vías. En el caso de los proyectos financiados por Fondecyt, la muestra se acotó a los estudios fi- nanciados dentro del grupo de Sociología y Ciencias de la Información, subdisciplina Ciencias de la Información. Además, se excluyó la línea de Fondecyt de Iniciación, pues su convocatoria es unipersonal (solo un Investigador Responsable), mientras que las líneas analizadas dan la posibilidad -no obligatoriade postular con equipos de investigación, figurando un Investigador Principal y Co-Investigadores. En síntesis, la muestra quedó conformada por un total de 57 proyectos: 34 correspondientes a Fondecyt Regular, y 23 a Estudios sobre Pluralismo en el sistema informativo nacional (anexo 1). Cabe indicar que la muestra se consideró solo hasta 2016 por la disponibilidad de acceso a la información de estos fondos al período de realización de la investigación.

Una vez tabulados los datos de cada uno de los 57 proyectos y los 417 artículos científicos, identificamos sus autores y afiliaciones institucionales. En base a esa información, se establecieron relaciones entre cada autor, considerando su género y número de trabajos elaborados de forma conjunta. Lo mismo se traduce a nivel de afiliación, considerando cantidad de proyectos y trabajos elaborados de forma inter-institucional. A continuación, las tablas se exportaron a un formato abierto (CSV) para ser procesadas con R Studio, un software libre que permite utilizar el lenguaje de estadística computacional $\mathrm{R}$, que permite el procesamiento de datos.

Utilizando RStudio, específicamente un paquete denominado IGRAPH, desarrollado para el análisis cuantitativo de redes y grafos, se importan los datos de los proyectos y los artículos científicos para identificar los actores e instituciones y crear cuatro redes para analizar: Para cada caso, proyectos y artículos, se crea una red de investigadores y otra de instituciones respectivamente. Posteriormente, mediante IGraph se efectuó el cálculo de los grados nodales, el diámetro y la densidad de la red.

Para la fase cualitativa -relacionada con el segundo de los objetivos- se trabajó con una muestra de 40 entrevistas aplicadas a investigadores e investigadoras de la Comunicación en Chile ( 22 hombres y 18 mujeres). La selección de los entrevistados se operacionalizó en el proyecto marco del que nace este artículo. Bajo la premisa de identificar la investigación para llegar a los investigadores, registramos el total de autores/as y co-autores/as con afiliación en institución chilena proyectos con financiamiento público (datos que también analizamos aquí en la fase cuantitativa), en comunicaciones presentadas en congreso nacional de la especialidad (de la Asociación Chilena de Investigadores en Comunicación -InCom), y en artículos publicados en revistas científicas de la especialidad chilenas y extranjeras indexadas en WOS. Con estas, obtuvimos un universo de 497 investigadores/ as con afiliación a instituciones chilenas. 
Para la fase cualitativa -aplicación de entrevistas- se configuró una muestra a través de la elaboración de un índice de ponderación del quehacer científico (anexo 2), para orientar la selección a aquellos investigadores/as que contaran con una trayectoria identificable y sostenida en el período de observación. Tras consulta con un panel de 12 expertos/as de la disciplina, se otorgó una ponderación a los distintos espacios de producción/difusión científica, asumiendo que cada una de estas actividades posee distintos grados de complejidad y dificultad. Con los valores obtenidos, se reordenó el universo de investigadores/as aplicando el valor ponderado a cada una de las actividades científicas registradas en el corpus de investigaciones. Además, se tomaron dos decisiones metodológicas: (a) acotar la población del estudio a aquellos investigadores/as que contaran con al menos dos actividades de producción/difusión del quehacer científico sistematizas $(U=467$, Población $=225)$; y (b) seleccionar como muestra al $20 \%$ de investigadores/as de la población con mayor valor de índice de ponderación de espacios de producción/difusión del quehacer científico, lo que derivó en 45 nombres. De estos, finalmente, se lograron realizar 40 entrevistas (a 22 hombres y 18 mujeres).

A esta muestra se aplicó un cuestionario de 29 preguntas (anexo 3), referidas tanto a su valoración del campo de la Comunicación en Chile en general, como a su propio quehacer científico en particular. En el caso de este trabajo se analizarán las preguntas 11 y 12 del cuestionario: (11) ¿Realiza su trabajo de investigación con otros/as académico/as? (12)¿Cómo se ha generado el trabajo conjunto o vínculo con estos investigadore/as?

El trabajo, procesamiento de la información obtenida con las entrevistas, se realizó a través de un análisis de contenido temático, basado en los principios de la teoría fundamentada (Glaser y Strauss 1967), donde se buscó identificar y categorizar las temáticas emergentes y sus significaciones o atributos asociados, a través de tres fases: codificación abierta de temáticas emergentes; re-organización semántica por afinidad conceptual de las temáticas emergentes; y re-codificación y configuración de un mapa de categorías conceptuales asociadas en (i) Categorías o temáticas principales identificadas como ámbitos de respuesta a las preguntas/objetivos de investigación; y (ii) Subcategorías o temáticas de afinidad conceptual que configuran una respuesta a la categoría principal.

\section{RESULTADOS: LOS ACTORES DE LA INVES- TIGACIÓN Y SUS REDES DE COLABORACIÓN}

La presentación de resultados la hemos dividido en dos fases. En la primera, nos centraremos en la descripción cuantitativa del análisis cienciométrico de las co-autorías. En la segunda, en los resultados cualitativos de las entrevistas.

Para el caso de los 417 artículos científicos, identificamos una participación de 497 investigadores/ as y de 169 instituciones. Analizando a los investigadores/as, encontramos una relación de 55\% investigadores hombres y $45 \%$ mujeres en su participación total en la red de investigación en comunicación. En los 57 proyectos concursables analizados, encontramos una participación de 96 investigadores/as y 21 instituciones. En el caso de los investigadores/as, encontramos una relación menos equilibrada, de 1 a 3, en la presencia de mujeres y hombres investigadores, tanto en su participación total, como en el rol de Investigadores Principales de los proyectos (Figura 2).

Figura 2. Relación investigadoras-investigadores

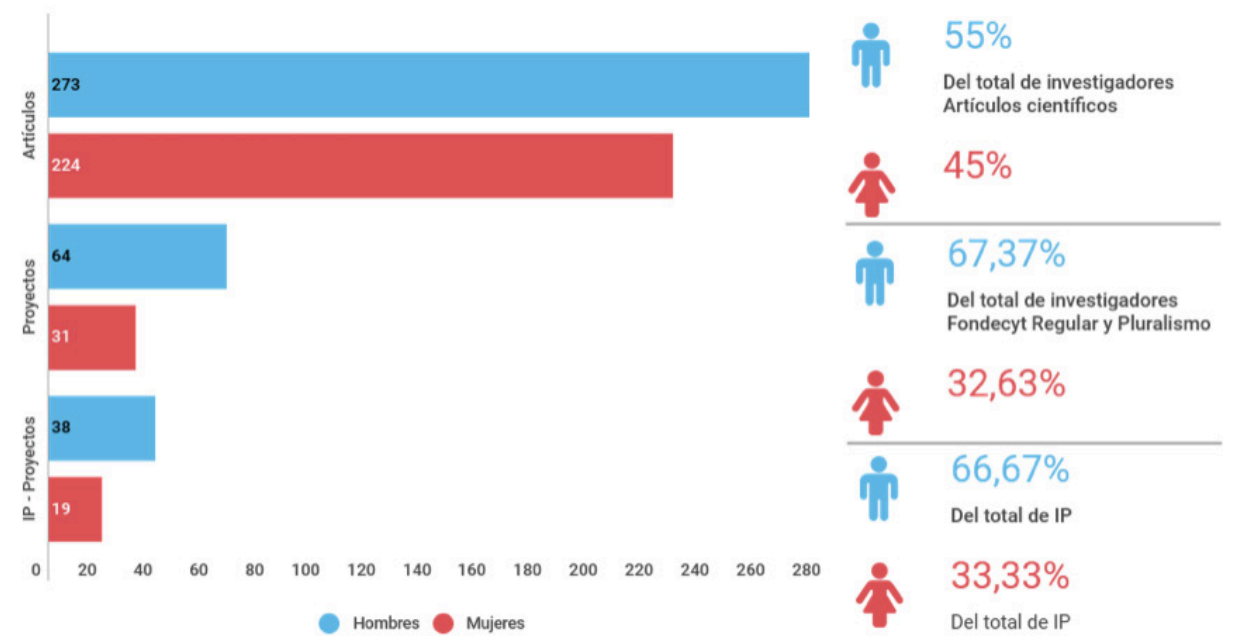


Una característica de la comunidad científica observada es su doblemente concentrada: tanto en las universidades con sede la Región Metropolitana (capital del país), como en dos centros de estudio principales que, en conjunto, representan el $25,3 \%$ del total de investigadores/as identificados en la muestra de proyectos analizados (Figura 3). Este datos que representamos a partir de los datos de participación en proyectos, se repite en el otro corpus analizado, y responde también al desarrollo histórico de la formación superior (universitaria) y al propio centralismo sociopolítico del país, que concentra la población y los polos políticos, económicos y sociales en el área capital.

En el análisis de red de co-autoría de los artículos científicos, se identifican un total de 497 investigadores con 695 relaciones entre ellos, lo que indica que la comunidad científica en Comunicación en Chile se caracteriza por una dispersión y escasos vínculos académicos. El análisis de la red de co-autoría de proyectos concursables, ofrece resultados similares: en el total de 96 autores que componen la red de colaboración académica en Comunicación en Chile, se observa un total de 149 relaciones entre ellos, lo que también es signo de dispersión en la red y pocos vínculos académicos. En resumen, para ambos grafos, más que una gran red de investigación, la red nos muestra un conjunto de subgrafos de diversos tamaños, inconexos entre sí.

Para el primer caso, al analizar los grados nodales de la red de investigadores/as en artículos científicos, identificamos que un grupo menor de actores fluctúan sus grados nodales entre 15 y 20 , mientras que la mayoría está por debajo de 5 e incluso con la presencia de algunos actores que presentan grado nodal 0 . Lo anterior implica que, si bien la red presenta muchos actores, los vínculos son escasos en relación al tamaño de la red.

Asimismo, si se analizan los grados nodales de cada uno de los actores de la red de proyectos concursables, se logran identificar solo cuatro (4) actores que tienen 10 o más nodos adyacentes; y 14 que presentan cero $(0)$ nodos adyacentes, lo que implica que, aunque integran el grafo, están aislados en la red chilena de investigación en Comunicación.

Por otra parte, el diámetro de la red de investigadores en artículos científicos, es decir, la distancia más grande entre un par de nodos, tiene un valor de 22. Considerando que la red está compuesta por un total de 497 actores, el diámetro es bajo, y esto se explica por la dispersión de sus actores,

Figura 3. Participación de investigadores/as e instituciones

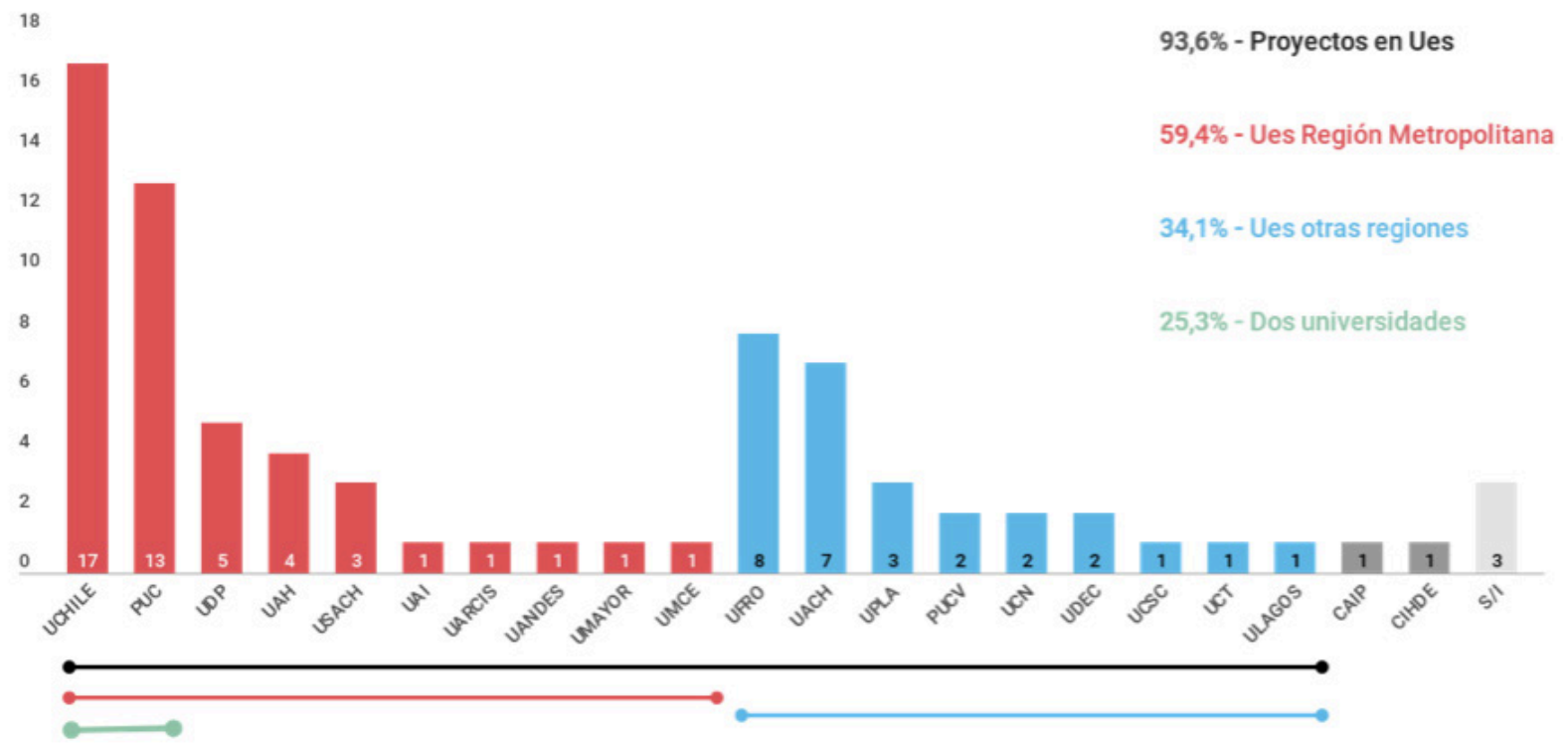

Eje Y, número de proyectos. Eje X, instituciones: UCHILE (U. de Chile), PUC (Pontificia Universidad Católica de Chile), UDP (U. Diego Portales), UAH (U. Alberto Hurtado), USACH (U. de Santiago de Chile), UAI (U. Adolfo Ibañez), UARCIS (U. de Artes y Cs. Sociales, en proceso de cierre), UANDES (U. de Los Andes), UMAYOR (U. Mayor), UMCE (U. Metropolitana de Cs. de la Educación), UFRO (U. de la Frontera), UACH (U. Austral de Chile), UPLA (U. de Playa Ancha), PUCV (Pontificia U. Católica de Valparaíso), UCN (U. Católica del Norte), UDEC (U. de Concepción), UCSC (U. Católica de la Santísima Concepción), UCT (U. Católica de Temuco), ULAGOS (U. de Los Lagos), CAIP (Centro de Análisis e Investigación Política), CIHDE (Centro de Investigaciones del Hombre en el Desierto), S/I (sin información). 
donde la gran cantidad de actores y la escasa cantidad de nexos hacen que el diámetro de la red no tenga un valor alto.

Para el caso de la red de proyectos, el diámetro tiene un valor de 6 y se explica de manera similar. Para un total de 96 nodos, el diámetro de la red resulta un valor bajo, al ser una red mayoritariamente dispersa. Debido a la existencia de varios subgrafos dentro de esta red, que no se encuentran relacionados entre sí, el diámetro tiende a ser bajo.

Al revisar la densidad ambas redes identificamos que tienen un valor de $0,6 \%$ y $33 \%$ aproximado respectivamente. Considerando que la densidad se entiende como la cantidad de vínculos que existen en la red, en relación a la cantidad de vínculos posibles, podemos identificar que resultan ser redes de investigación poco densas, ya que sus actores no colaboran de forma significativa entre ellos, o lo hacen de forma muy parcial.

A grandes rasgos, si se visualizan los indicadores de grado nodal, diámetro y densidad de red, se corrobora lo que indican las gráficas: la red de investigación en Comunicación en Chile se resume en un conjunto de grupos de investigación aislados que colaboran puntualmente entre ellos, donde no más de 10 actores se llevan el protagonismo en colaboración. La fragmentación de estas redes también se debe a que el $14.6 \%$ de los actores no presenta ningún tipo de colaboración científica y han presentado sus trabajos de manera aislada.

En el tema de género, el grado de artículos científicos se muestra mucho más equilibrado (Figura 4), existiendo una participación del $55 \%$ de hombres en color azul y el $45 \%$ de mujeres en color rojo. A diferencia de lo anterior, la Figura 6 de proyectos concursables muestra 32 nodos de color rojo, que indican la cantidad de investigadoras que integran la red $(33.3 \%)$, y 64 nodos de color azul, que indica la cantidad de investigadores. Lo anterior indica un claro predominio del género masculino en la red, duplicando al género femenino. Sin embargo, el $90.6 \%$ de las mujeres participa con algún tipo de colaboración científica, mientras que solo el $9.4 \%$ trabaja de manera aislada. En el caso de los hombres, el $82.8 \%$ presenta alguna co-autoría, mientras que el $17.2 \%$ de ellos trabaja de manera aislada. En una relación proporcional, es posible afirmar que el género femenino trabaja de manera más colaborativa que el género masculino.

Las Figuras 5 y 7 nos muestran la red de colaboración científica institucional chilena en el ámbito de la comunicación, considerando la muestra de artículos científicos y de proyectos respectivamente.

La primera red muestra un total de 169 instituciones y 444 relaciones entre ellas. Por otro lado, la segunda red está compuesta por 21 instituciones y 17 relaciones entre ellas.

Cuando visualizamos los grados nodales de la red de artículos, identificamos que las instituciones que tienen mayor grado son la Universidad de Santiago, con un valor de 33, y la Pontificia Universidad Católica de Valparaíso, con un valor de 31. Ambas instituciones no resultan ser las más productivas de la red, como la Universidad de Chile o la Pontificia Universidad Católica, pero son

Figuras 4 y 5. Relaciones entre investigadores/as e instituciones en artículos científicos
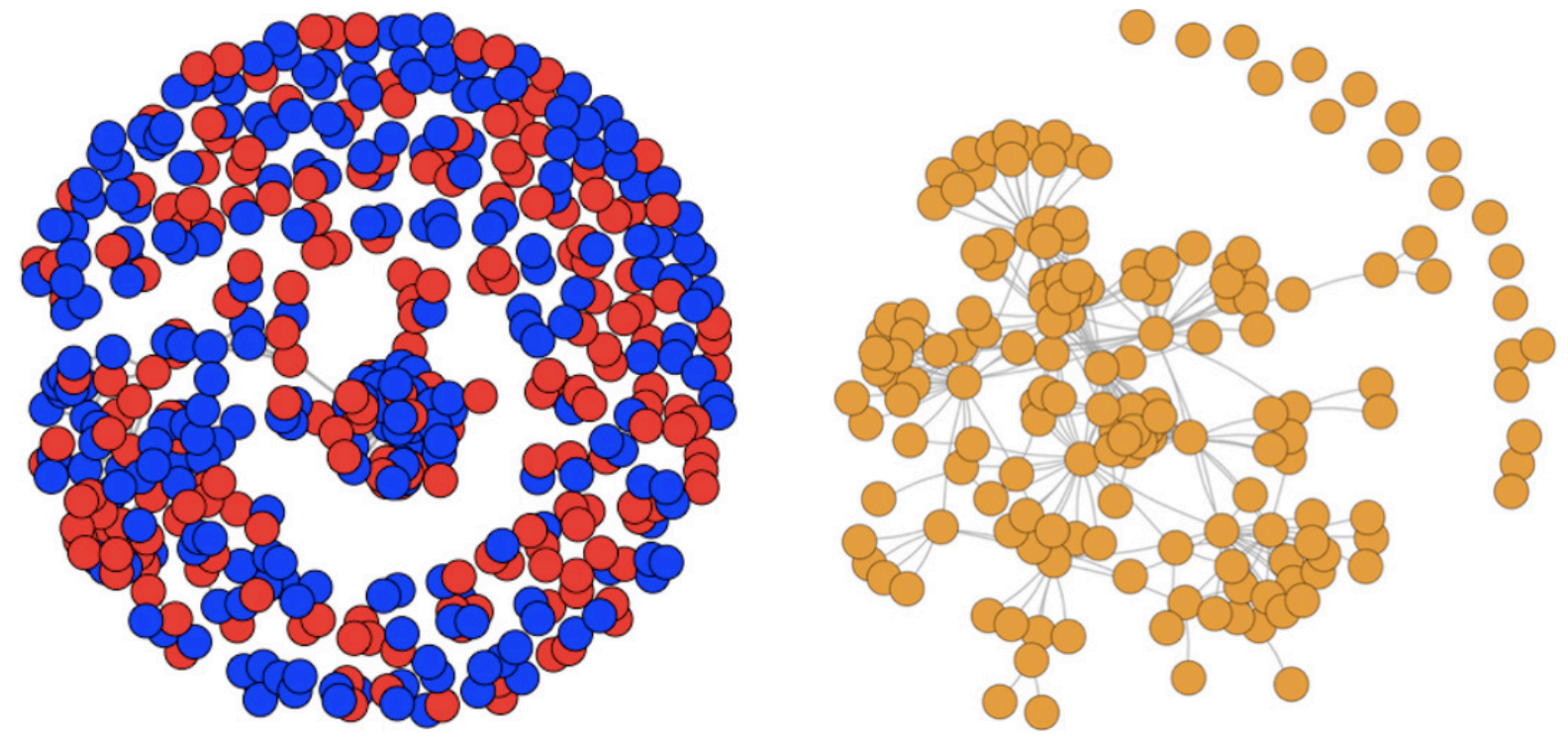
Figuras 6 y 7. Relaciones entre investigadores/as e instituciones en proyectos concursables

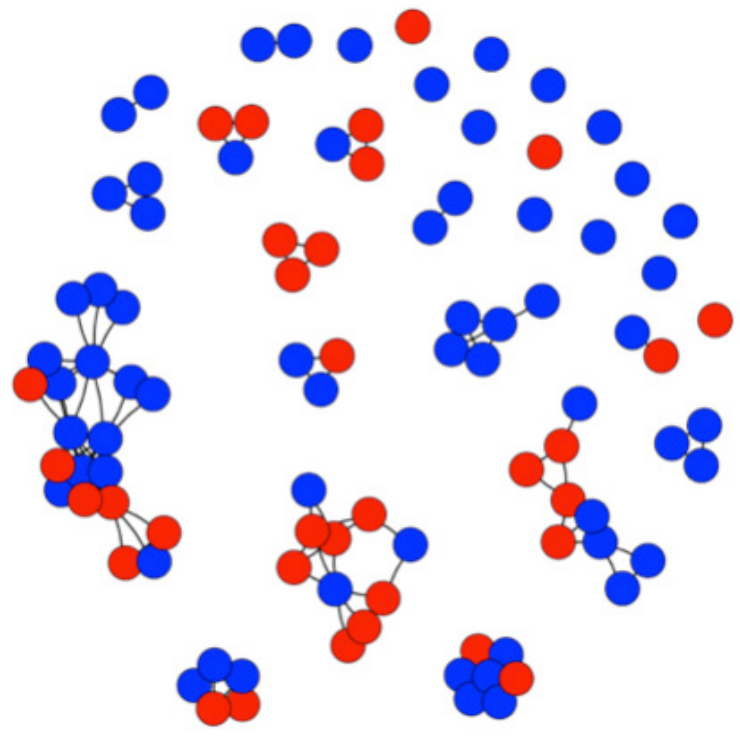

las que más vínculos generan con otras instituciones para desarrollar sus publicaciones científicas y, por tanto, sus investigaciones no son endogámicas. Nuestro conocimiento de la comunidad científica analizada, nos permite contextualizar este dato, pues más que una tendencia institucional a la colaboración académica por parte de las primeras (Universidad de Santiago y Pontificia Universidad Católica de Valparaíso), el fenómeno se explica por el rol de una investigadora en particular (Dra. Claudia Mellado Ruiz), de afiliación a ambas entidades en momentos temporales diferentes, y que desde hace unos años lidera un proyecto internacional sobre roles periodísticos, en el que participan cerca de 40 países. A diferencia de lo anterior existen 14 actores que presentan grado nodal 0 , es decir, no presentan ningún tipo de colaboración.

Por otra parte, al analizar los grados nodales de todas las instituciones participantes en la red de proyectos concursables, destaca la participación de la Universidad de Chile como el actor con un mayor grado nodal, con un valor 8 , y con la segunda mayor productividad de proyectos, es decir, resulta la institución más colaborativa en la red. A diferencia de la Pontificia Universidad Católica, que resulta la institución más productiva, pero su grado nodal es 1 , es decir, su nivel de colaboración resulta bajo. Por otra parte, el $42,86 \%$ de las instituciones participantes de la red no presenta ningún tipo de colaboración y trabajan de forma aislada, lo que refleja una carencia significativa en la colaboración inter-institucional a nivel nacional.

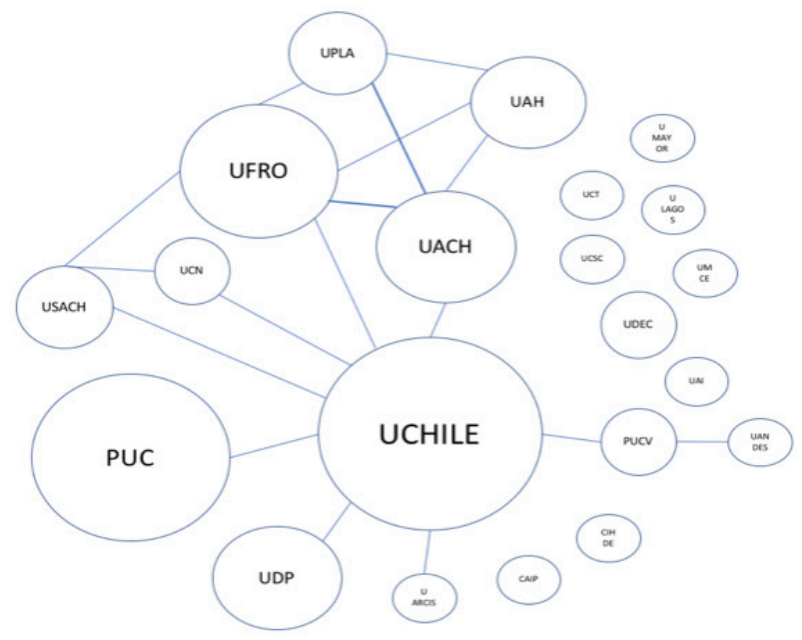

UCHILE (U. de Chile), PUC (Pontificia Universidad Católica de Chile), UDP (U. Diego Portales), UAH (U. Alberto Hurtado), USACH (U. de Santiago de Chile), UAI (U. Adolfo Ibañez), UARCIS (U. de Artes y Cs. Sociales, en proceso de cierre), UANDES (U. de Los Andes), UMAYOR (U. Mayor), UMCE (U. Metropolitana de Cs. de la Educación), UFRO (U. de la Frontera), UACH (U. Austral de Chile), UPLA (U. de Playa Ancha), PUCV (Pontificia U. Católica de Valparaíso), UCN (U. Católica del Norte), UDEC (U. de Concepción), UCSC (U. Católica de la Santísima Concepción), UCT (U. Católica de Temuco), ULAGOS (U. de Los Lagos), CAIP (Centro de Análisis e Investigación Política), CIHDE (Centro de Investigaciones del Hombre en el Desierto)

Al visualizar el diámetro de la red de artículos científicos identificamos que su valor es 8 de un total de 169 vértices. Asimismo, es posible visualizar el diámetro en la distancia máxima de la red entre los actores "U Erasmus de Rotterdam" y "U Central", haciendo el siguiente recorrido: U Erasmus de Rotterdam - U Alberto Hurtado - U Adolfo Ibáñez - U Chile - PU Católica - U Santo Tomás - U Católica de la Santísima Concepción - U Central. En el caso de los artículos, sobre todo en los publicados en revistas internacionales, se observa una mayor co-autoría con investigadores de instituciones extranjeras, a diferencia de las revistas nacionales donde lo predominante es la co-autoría con investigadores de la propia institución nacional. A nivel de hipótesis, observamos que esta tendencia pudiese explicarse y contextualizarse en los procesos formativos de postgrados de los investigadores, pues las mayores relaciones inter-institucionales corresponden, efectivamente, a los espacios académicos de especialización doctoral.

El diámetro de la red de proyectos tiene un valor de 5 , siendo visible en la distancia máxima de la 
red, entre los vértices UAH (U. Alberto Hurtado) y UANDES (U. de Los Andes), haciendo el siguiente recorrido: UAH (U. Alberto Hurtado)- UACH (U. Austral de Chile) - UCHILE (U. de Chile) - PUCV (Pontificia U. Católica de Valparaíso) - UANDES (U. de Los Andes). El diámetro de la red resulta pequeño debido a la fragmentación de la red y a la participación de nodos aislados, que no presentan ningún tipo de colaboración con las otras instituciones.

Por otra parte, como se ha indicado previamente, estas redes inter-institucionales, tanto de artículos como de proyectos concursables, presentan más nodos que relaciones entre ellos, lo cual habla de redes con escasa densidad. El cálculo de la cantidad de nodos existentes en relación a la cantidad de nodos posibles, indica que la red de artículos científicos posee un 3\% de densidad y la red de proyectos concursables posee un $8 \%$ de todas las potenciales colaboraciones que podrían existir entre estas instituciones.

De lo anterior, se desprende que las instituciones más colaborativas en la red chilena de investigación en Comunicación resultan ser la Universidad de Santiago y la Pontificia Universidad Católica de Valparaíso, en el caso de los artículos científicos, y la Universidad de Chile en el caso de los proyectos de investigación concursables. En términos generales, las redes de autoría e institucionales demuestran una comunidad de investigación nacional en Comunicación fragmentada, debido a la escasa densidad de las relaciones entre los actores.

\section{Las significaciones del trabajo colaborativo}

Junto a esta mirada cuantitativa, creemos interesante complementar estos datos con la valoración subjetiva de los propios investigadores/as. Si bien comprendemos que sus respuestas no son generalizables, pues se trata de la perspectiva subjetiva de los entrevistados, revelamos el interés de sus respuestas y significaciones por tratarse de pro- tagonistas activos de la comunidad científica de la Comunicación en Chile.

A partir de un análisis de contenido temático, identificamos tres categorías centrales (Motivaciones para el trabajo en red; Condiciones para el trabajo en red; Potencialidades del trabajo en red) con sus respectivas subcategorías (Figura 8 ) y relaciones.

Si observamos la frecuencia de respuestas, un tercio de la muestra caracteriza su trabajo de investigación de manera colaborativa. Incluso aquellos que indican alguna preferencia por el trabajo individual, reconocen su aprendizaje del trabajo en red, ya sea por motivaciones externas (requisitos de un espacio de investigación específico), personales, o por la necesidad de un objeto o proyecto de investigación particular. En definitiva, se prefiera o no de manera inicial, sí es posible identificar consenso sobre la relevancia del trabajo colaborativo.

Si consideramos las Motivaciones para el trabajo en red, de las entrevistas se desprenden tres tendencias principales: afinidad temática, necesidad interdisciplinaria y afinidad personal. Entre estas, el factor más determinante serían las relaciones personales, muy por encima de las otras subcategorías. De hecho se puntualiza que si bien la afinidad temática puede ser el punto de inicio de un trabajo colaborativo, solo el desarrollo de vínculos personales -de amistad incluso- garantizarán la consolidación del trabajo en red y, en definitiva, la formación de un equipo de trabajo, que se perpetúe más allá de una colaboración puntual ["Las redes más fuertes, las que continúan siendo más o menos estables, son las que nacen a partir de intereses comunes y uno genera más un vínculo de amistad que un vínculo de trabajo, esas son las que permanecen" (Entrevistada 8)].

Las colaboraciones puntuales -la escritura de un paper, por ejemplo- pueden nacer de intereses comunes o de la necesidad de complementar

Figura 8. Mapa de categorías temáticas sobre valoración virtuosa del trabajo en red

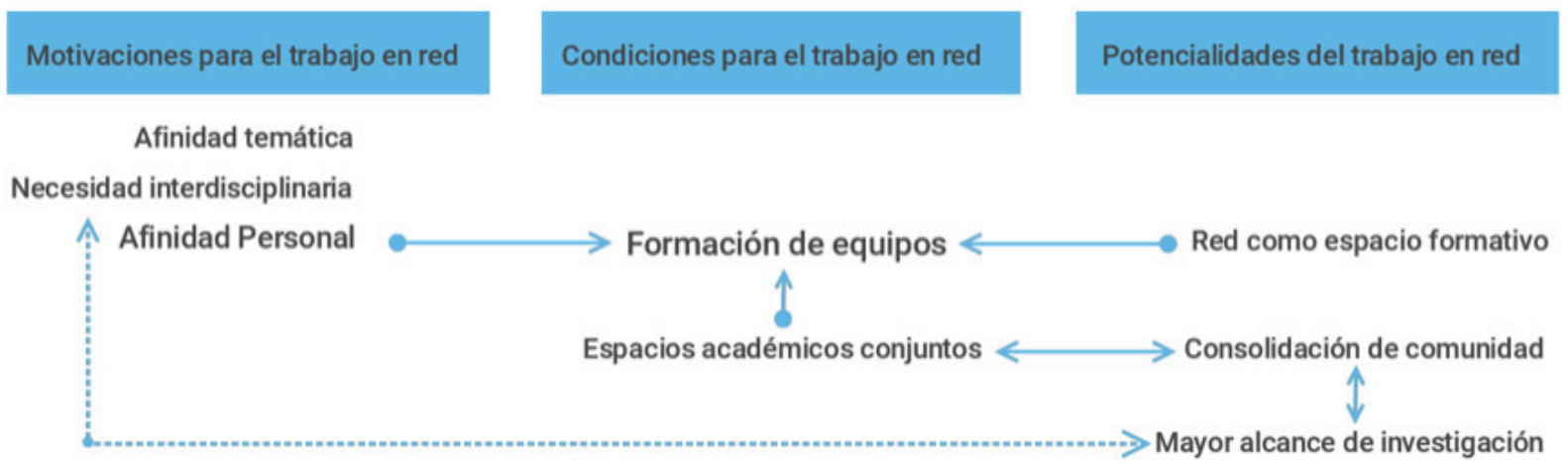


interdisciplinariamente el abordaje de un tema, lo que no implica necesariamente trabajo colaborativo o en red permanente ["Sé que hay gente que no necesita sentirse cómodo, pero si yo voy a trabajar contigo, necesito pasar una hora y media tomándome un café. Si no soy capaz de tomarme un café, entonces da para que escribamos un paper juntas, pero no da para un trabajo de largo aliento como un Fondecyt" (Entrevistada 17)].

Al revés, en tanto, la afinidad personal sí se valora con mayor densidad como punto de inicio del trabajo colaborativo: desde una buena relación personal, con un espacio de simpatía y confianza recíproca, pueden surgir ideas compartidas para el desarrollo de investigaciones conjuntas. En cambio, a pesar de que exista afinidad temática, si no se logra un fiato personal inicial, no se desarrollará en trabajo colaborativo ["Afinidad temática sí he tenido con otras personas pero que no hemos logrado plasmar en trabajos concretos. Principalmente ha sido un tema de mucho 'fiato' como grupo, de mucha confianza porque a la larga estás pensando en una labor que te va a tomar cuatro años, o peor como en el caso de los proyectos sobre pluralismo, donde en nueve meses debes rematar todo, por lo que necesitas saber que el otro va a poner al servicio toda su capacidad, todo su esfuerzo" (Entrevistado 19)].

Este elemento, nos vincula a una de las subcategorías del nodo Condiciones para el trabajo en red: la formación de equipos. Puede leerse como una explicación tautológica: para el trabajo colaborativo se requiere un equipo de trabajo, pero esa evidencia nos puede permitir comprender la articulación de nuestra propia comunidad: su concentración.

La formación de un equipo de trabajo requiere tiempo: la generación de confianza y lazos personales -ya reseñamos su centralidad como motivación- solo se facilita a través del contacto permanente y cotidiano, lo que se ve facilitado en el espacio intra-universitario, ya sea por el contacto laboral simétrico -colegas o compañeros de trabajo- o por haber compartido instancias formativas previas como compañeros de carrera, relación estudiante-profesor, por ejemplo ["Mis redes de trabajo tienen harto que ver con mis afectos, yo trabajo con gente con la que me formé, ya sea porque fueran mis profesores o fuimos compañeros o fueron mis estudiantes y ahora son colegas. Es bien endogámica, si se quiere, y se ha ido abriendo en la medida que he ido conociendo o he podido establecer otras redes con otros académicos, pero también son difíciles esas conexiones (...) en qué momento uno se encuentra, en qué momento uno construye proyectos, ideas de publicación. Eso requiere tiempo" (Entrevistada 7)].
En ese sentido, se comprende esta concentración de las redes evidenciada en la fase cuantitativa, pues todas derivan de equipos de trabajo formados en comunidades académicas específicas. Incluso, las redes no concentradas intra-universidad, se explican también por este mismo factor, pues corresponden a investigadores que comparten la formación de postgrado $y$, por ende, una determinada perspectiva teórica o interpretativa la que, observamos desde la experiencia de los propios entrevistados, se fortalece y desarrolla precisamente a través del trabajo conjunto. En este sentido, estaríamos frente a un círculo endogámico virtuoso, en el sentido que deriva en la consistencia de un trabajo de investigación sostenido en el tiempo y que, podemos inferir, de mayor especialización de los equipos. En contrapartida, sin embargo, podría tener una arista más negativa, si asumimos como positivo el contacto inter-universitario. Esta afirmación se desprende también de los propios investigadores, quienes indican que valorarían positivamente la posibilidad de trabajar en red con investigadores de otras universidades, pero esto se dificulta, precisamente, por la escasa posibilidad temporal para trabajar en formación de equipos de trabajo. Esto, asumiendo, como ya hemos dicho, que la formación de equipos, la configuración de redes colaborativas, es más que el trabajo conjunto puntual.

En esta misma línea, los investigadores sí identifican como posible solución el fortalecimiento de espacios académicos conjuntos, que fomenten el encuentro entre investigadores, mayoritariamente los congresos, valorados en cuanto espacio presencial que, por una parte, permiten conocer el trabajo que llevan a cabo otros académicos (vinculado a la afinidad temática) pero, sobre todo, generar espacios para el conocimiento personal con investigadores que la cotidianidad del espacio laboral no facilita encontrar (vinculado a la afinidad personal).

En este sentido, como punto referente clave, los investigadores destacan la formación de la Asociación Chilena de Investigadores en Comunicación -InCom- y, en especial, la realización de su congreso anual, que en 2018 realiza su quinta versión, como un paso clave y fundamental para fomentar la configuración y consolidación de una comunidad científica de la Comunicación en Chile, conocida y articulada, que, eventualmente podría redundar en el trabajo colaborativo ["InCom en ese sentido es súper importante $y$ todo lo que podamos hacer en términos locales para hacer más tupidas esas redes, es súper importante. Había una sensación de rivalidad que se ha ido superando con el paso de los años, y que es impresionante como hoy día tú conoces gente de, prácticamente, todas las universidades que están haciendo investigación en Chile, 
con pocas excepciones de investigadores que no les interesa" (Entrevistada 5)].

Sobre este punto, dos acentos específicos: por una parte, reiterar el elemento basal de las relaciones personales como punto de inicio para el trabajo colaborativo y, luego, la formación de equipos y, por otra, el aporte de estos espacios para la consolidación de una comunidad científica en cuanto a su reconomiento en la estructura de la política pública del país. Como contexto, en Chile los estudios sobre comunicación no cuentan con un grupo de estudio autónomo en la Comisión Nacional de Ciencia y Tecnología -principal organismo estatal de apoyo y financiamiento de la investigación en el país- sino que es parte, como subdisciplina (Ciencias de la Comunicación e Información), del grupo de Sociología y Ciencias de la Información, junto a Cambio Social y Desarrollo, Metodología, Sociología de las Organizaciones Formales, Sociología Urbana y Rural, Teoría Socio-Cultural, Sociología del Trabajo, Otras Sociologías, Política Científica y Tecnológica, y Trabajo Social.

En esta misma línea, y en cuanto a las potencialidades del trabajo en red, se destaca como la posibilidad de avanzar en la consolidación de la disciplina por medio del fortalecimiento de una comunidad científica, que trascienda el espacio intra-universitario. Es decir, además, de asegurar el mayor alcance del trabajo de investigación a través de abordar un fenómeno con mayor complejidad y profundidad de variables, tanto geográfico (estudios comparativos a través de redes internacionales), disciplinario (ya nos hemos referido a la interdisciplina como una de las motivaciones para el trabajo en red), como también en la profundidad de enfoques desde la misma disciplina, para lo que sería clave la articulación de equipos de trabajo ya existentes en las mismas universidades, lo que en doble vínculo sería indicio de una consolidación de la comunidad científica y aportaría al fortalecimiento de la misma consolidación ["Me parece que es el momento también para empezar a fortalecer las redes, o sea no trabajar con el círculo como "más íntimo" sino que empezar a colaborar con otros. Podemos empezar a desarrollar proyectos de mayor envergadura que permitan que comunicaciones/periodismo se tomen como una disciplina en serio" (Entrevistada 4)].

Finalmente, algunos investigadores (aunque solo cuatro menciones), destacan las potencialidades del trabajo colaborativo como un espacio formativo para integrar a la experiencia de investigación a estudiantes de pre y postgrado, elemento -quizáinicial de su futura incorporación, en caso de optar por la carrera académica, a equipos de trabajo en su propia universidad de formación (subcategoría formación de equipos).

\section{CONCLUSIONES}

En una era de hiperconectividad, en que internet y el ecosistema tecnológico facilita el acceso a información y nos brinda la posibilidad de inmediatizar el contacto con colegas en distintas latitudes (dentro y fuera del país), nos encontramos frente al escenario ideal para cultivar el trabajo colaborativo. Terreno fértil para el desarrollo de las co-autorías, tanto en la generación como en la difusión de nuevo conocimiento, que sintonizaría, además, con la necesidad de desarrollar un trabajo académico "productivo" y cada vez más exigente y competitivo: dividir y optimizar esfuerzos, miradas y tiempos para aumentar los indicadores de postulación a proyectos y redacción de artículos académicos. Como ya hemos citado, asociarse o perecer (Aguado-López y otros, 2018) podría ser una máxima de estos tiempos en la academia.

Pero además de esta dimensión cuantitativa -cuando no estratégica- el trabajo colaborativo, permite complejizar también la aproximación a los fenómenos estudiados aportando densidad teórica, interdisciplinariedad e incluso garantías de calidad al no restringirse el texto a la mirada de un único autor. De hecho, un estudio reciente realizado entre investigadores de la Comunidad Valenciana, señala que entre las motivaciones para el desarrollo de actividades de investigación en colaboración, las primeras preferencias se orientan a 'aprovechar las habilidades, destrezas o conocimientos de los colaboradores' y a la 'aproximación multidisciplinar al tema estudiado', por sobre la opción de 'incrementar la productividad' que se ubica en el tercer lugar (Gómez-Ferri y González-Alcaide, 2018). Así, entonces, podemos asumir o interpretar que el trabajo colaborativo sería más diálogo académico, que solo una suma de fragmentos.

Con esto, nos posicionamos desde un punto de vista favorable hacia el trabajo colaborativo. Sin embargo, a partir del trabajo realizado, situado en una comunidad científica particular, quisiéramos poner algunos acentos.

Entre estos, la dimensión central del factor personal como elemento fundamental del trabajo colaborativo. En ese sentido creemos, como Gómez-Ferri y González-Alcaide (2018) "que la colaboración científica y las formas que adopta no sería un mero subproducto de la interiorización de normas o reglas de la institución, sino una creación activa de los propios sujetos del campo"

En este sentido, resulta interesante que uno de los valores esenciales para el trabajo colaborativo traspasa las competencias técnicas y profesionales -quizás porque en el nivel académico se asumen como pre-existentes- sino que es la valoración per- 
sonal el factor predominante. Así queda patente desde nuestros resultados, los que a pesar se ser situados y acotados, son coherentes con hallazgos similares en otras comunidades académicas, como el ya citado estudio de Gómez-Ferri y González-Alcaide (2018) en la Comunidad Valenciana, quienes también identifican, de las voces de investigadores e investigadoras, que "la generación de un clima de confianza, conocimiento y respeto mutuo es el rasgo personal más valorado por parte de los investigadores para el desarrollo de actividades de investigación en colaboración".

Resulta interesante, sin embargo, que la generación de confianzas interpersonales pareciera ir a contracorriente de las exigencias de la productividad académica, más tendiente a la competencia (por fondos, por espacios de publicación) que a la colaboración. La misma característica de las actuales condiciones y exigencias de la carrera universitaria puede comprenderse en tensión con el desarrollo del trabajo colaborativo. Por una parte, un aliado: mayor colaboración, mayor producción. Por otra, un desincentivo para el mismo. O más bien para la conformación y consolidación de nuevos equipos. Como ya hemos visto, esta sería una de las explicaciones de las redes intra-universitarias por sobre las inter-universitarias, pudiendo asumir las primeras como más endogámicas y menos diversas (mayor tendencia a compartir perspectivas teóricas, en especial cuando se comparte formación inicial de pregrado o postgrado), pues no habría tiempo para el desarrollo ni conformación de equipos de trabajo permanentes y que pudieran proyectar líneas de investigación comunes.

En el caso de esta investigación, asumimos sus limitaciones al tratarse de una mirada acotada disciplinaria y geográficamente. Disciplinariamente, tanto el corpus cuantitativo como cualitativo se ha restringido a aquello definido estrictamente como ciencias de la información y la comunicación. Esta decisión ha sido coherente con el proyecto de investigación que le da marco y que, como hemos indicado, tiene como propósito el conocimiento denso de la comunidad académica dedicada a la investigación en Comunicación en Chile. De esta manera, no se han abordado espacios académicos fronterizos e interdisciplinarios, los que asumimos como necesarios para una comprensión más compleja del área, en especial por las propias características de la propia disciplina. Sin embargo, y ya habiendo indicado sus debilidades, sí creemos que la mirada específica a una comunidad científica particular, y en especial a través de la combinación de técnicas cuantitativas y cualitativas, es posible aportar desde las voces de los propios investigadores e investigadoras algunos elementos más para comprender las motivaciones, sentidos y significaciones que dotan de subjetividad una forma ya instalada en la producción académica, como lo es el trabajo en redes de colaboración.

En el contexto chileno donde hemos situado este trabajo y de cuya comunidad científica somos parte, resulta interesante que de manera predominante, los investigadores/as entrevistados, muchos pertenecientes a los nodos concentrados de generación de investigación, manifiestan como un deseo avanzar hacia la conformación de redes más abiertas y diversas. $Y$ del deseo, al hecho, pues han sido promotores y actores del hito clave de articulación del campo en Chile: la Asociación Chilena de Investigadores en Comunicación -InCom-, que como asociación y congreso, se valoran como el principal espacio de encuentro de la comunidad científica nacional. Más allá de la teoría, desde ese lugar colectivo han nacido monográficos con editores inter-universitarios y seminarios colaborativos. En definitiva, y si la tendencia se mantiene y el proyecto como hasta ahora, es probable que en plazos más breves que largos, la dispersa y fragmentada red que hemos graficado, avance hacia una red de intelectuales, masa crítica de una comunidad científica consolidada. Por ahora, habrá que esperar. Esperar y colaborar.

\section{AGRADECIMIENTOS}

Este trabajo ha sido financiado por la Comisión Nacional de Ciencia y Tecnología -Conicyt- a través del proyecto"Investigación en Comunicación en Chile: estado del arte y análisis de las características y proyecciones de la comunidad científica nacional" [COD Fondecyt 11160842].

* La Comisión Nacional de Investigación Científica y Tecnológica -Conicyt- desde el 1 de enero de 2020 cambió su nombre a Agencia Nacional de Investigación y Desarrollo -Anid-.

\section{ACKNOWLEDGEMENTS}

This work has been funded by the National Commission of Science and Technology -Conicytthrough the project "Research in Communication in Chile: state of the art and analysis of the characteristics and projections of the national scientific community" [COD Fondecyt 11160842].

* La Comisión Nacional de Investigación Científica y Tecnológica -Conicyt- since january 2020 is called Agencia Nacional de Investigación y Desarrollo -Anid-. 


\section{REFERENCIAS}

Aguado-López, E.; Becerril-García, A.; Godínez-Larios, S. (2018). Asociarse o perecer: la colaboración funcional en las ciencias sociales latinoamericanas. Revista Española de Investigaciones Sociológicas, 161, 3-22.

Caldevilla D.; Del Valle C. (2011). Análisis de las trayectorias investigadoras de las mujeres chilenas a partir de sus publicaciones científicas en idioma español (I parte). Madrid: Visión Libros.

Casas-Roma, J.; Pérez-Solà, C. (2016). Análisis de datos de redes sociales. Barcelona: Editorial UOC.

Carrasco, E. (2013). La unidad faltante, especificidad y rol de las humanidades. Revista Chilena de Literatura, 84, 19-36. https://doi.org/10.4067/S071822952013000200003

Conicyt - Comisión Nacional de Investigación Científica y Tecnológica (2019). Sitio web institucional. https:// www.conicyt.cl

Devés, E. (2004). La circulación de las ideas y la inserción de los cientistas económico-sociales chilenos en las redes conosureñas durante los largos 1960. Historia, 37(2), 337-366.

Dittus, R. (2008). Cartografía de los Estudios mediales en Chile. Concepción: Ediciones Universidad Católica de la Santísima Concepción.

Edwards, P.; Mayernik, M.; Batcheller, A.; Bowker, G.; Borgman, C. (2011). Science friction: Data, metadata, and collaboration. Social Studies of Science, 41, 667690. https://doi.org/10.1177/0306312711413314

Escribà, E.; Cortiñas, S. (2013). La internacionalización y las coautorías en las principales revistas científicas de Comunicación en España. Comunicar [en linea], 41, 3544.

Finholt, T. A.; Olson, G. M. (1997). From Laboratories to Collaboratories: A New Organizational Form for Scientific Collaboration. Psychological Science, 8(1), 28-36. https://doi.org/10.1111/j.1467-9280.1997.tb00540.x

Glaser, B.; Strauss, A. (1967). The Discovery of Grounded Theory: strategies for qualitative research. Chicago: Aldine. https://doi.org/10.1097/00006199196807000-00014

Gómez-Ferri, J.; González-Alcaide, G. (2018). Patrones y estrategias en la colaboración científica: la percepción de los investigadores. Revista Española de Documentación Científica, 41 (1): e199. https://doi.org/10.3989/ redc. 2018.1.1458

González Alcaide, G.; Gómez Ferri, J. (2014). La colaboración científica: principales líneas de investigación y retos de futuro. Revista Española de Documentación Científica, 37(4): e062. https://doi.org/10.3989/redc.2014.4.1186

Jung, N.; Ruiz-León, A. A. (2018). Lo local y lo global de la colaboración científica: ¿qué significa, y cómo visualizarlo y medirlo? Revista Española de Documentación Científica, 41 (2): e203. https://doi.org/10.3989/ redc. 2018.2 .1463
Katz, J. S.: Martin, B. R. (1997). What is research collaboration? Research Policy, 26(1), 1-18. https://doi. org/10.1016/S0048-7333(96)00917-1

Kuhn, T. (1975). La estructura de las revoluciones científicas. México D.F.: Fondo de Cultura Económica S.A.

Lagos, C. (2018). Theoretical Frames and Institutional Constraints: A Synopsis about Chilean Communication Research in the 21st Century. International Journal of Communication, 12, 3253-3273.

Lazcano-Peña, D. (2007). Investigación en Comunicación Social en Chile: configurando el campo nacional. En: Santander, P. (ed.), Los medios en Chile: voces y contextos, pp. 121-147. Valparaíso: Ediciones Universitarias de Valparaíso S.A.

Lazcano-Peña, D.; Perry, A. (2016). Investigación en comunicación en Chile: un mapa de su apoyo público, y la evidencia de su concentración. Anuario Electrónico de Estudios en Comunicación Social "Disertaciones", 9 (2), 92-116. https://doi.org/10.12804/disertaciones.09.02.2016.05

López-Borrull, A.; Canals, A. (2013). La colaboración científica en el marco de nuevas propuestas científicas: OpenScience,e-ScienceyBigData. En González Alcaide, G.; Agulló Calatayud, V., Gómez Ferri, J. (eds.), La colaboración científica: una aproximación multidisciplinar, pp. 91-100. Nau Llibres, Edicions Culturals Valencianes, S.A.

Martínez Nicolás, M.; Saperas Lapiedra, E. (2011). La investigación sobre Comunicación en España (1998-2007). Análisis de los artículos publicados en revistas científicas. Revista Latina de Comunicación Social, 66, 101-129. https://doi.org/10.4185/RLCS-66-2011-926-101-129

Martínez Nicolás, M.; Saperas Lapiedra, E. (2016). Objetos de estudio y orientación metodológica de la reciente investigación sobre comunicación en España (2008-2014). Revista Latina de Comunicación Social, 71, 1365-1384. https://doi.org/10.4185/RLCS-2016-1150es

Newman, M. E. J. (2004). Coauthorship networks and patterns of scientific collaboration. Proceedings of the National Academy of Sciences of the United States of America, 101(SUPPL. 1), 5200-5205. https://doi. org/10.1073/pnas.0307545100

Price, D. (1963). Little science, big science. New York: Columbia University Press. https://doi.org/10.7312/ pric91844

Price, D.; Beaver, D. (1966). Collaboration in an invisible college. American Psychologist, 21 (11), 1011-1018. https://doi.org/10.1037/h0024051

Sonnenwald, D. H. (2007). Scientific collaboration. Annual Review of Information Science and Technology, 41, 643681. https://doi.org/10.1002/aris.2007.1440410121

Todeschini, R.; Baccini, A. (2016). Handbook of Bibliometric Indicators: Quantitative Tools for Studying and Evaluating Research. New Jersey: John Wiley \& Sons. https://doi.org/10.1002/9783527681969 
Valle, C. del (2004). Metainvestigación de la comunicación en Chile. Tendencias y crítica. Temuco: Ediciones Universidad de la Frontera.

Vassallo de Lopes, M. (2001). Reflexiones sobre el estatuto disciplinario del campo de la comunicación. En: Vassallo de Lopes, M; Fuentes Navarro, R. (coords.), Comunicación: Campo y Objeto de Estudio. Perspecti- vas Reflexivas Latinoamericanas, pp. 43-58. Tlaquepaque: Instituto Tecnológico y de Estudios Superiores de Occidente (ITESO)

Wasserman, S.; Faust, K. (1994). Social Network Analysis: Methods and Applications. Cambridge: Cambridge University Press. https://doi.org/10.1017/ CBO9780511815478

\section{ANEXOS}

Anexo 1. Corpus de análisis fase cuantitativa

\begin{tabular}{|c|c|c|c|c|}
\hline & $\begin{array}{c}\text { Fondecyt } \\
\text { Regular }\end{array}$ & $\begin{array}{l}\text { Estudios sobre } \\
\text { Pluralismo en el } \\
\text { sistema informa- } \\
\text { tivo nacional }\end{array}$ & $\begin{array}{c}\text { Artículos publicados en revistas nacionales } \\
\text { (Cuadernos.info; Comunicación y Medios, } \\
\text { F@ro: Perspectivas de la Comunicación, } \\
\text { Tercer Milenio) }\end{array}$ & $\begin{array}{l}\text { Artículos publicados } \\
\text { en revistas interna- } \\
\text { cionales indexadas } \\
\text { en base de datos } \\
\text { WOS }\end{array}$ \\
\hline 2010 & 3 & 2 & 33 & 10 \\
\hline 2011 & 9 & 3 & 30 & 20 \\
\hline 2012 & 3 & 2 & 32 & 14 \\
\hline 2013 & 3 & 4 & 27 & 12 \\
\hline 2014 & 4 & 4 & 20 & 16 \\
\hline 2015 & 8 & 4 & 21 & 37 \\
\hline 2016 & 4 & 4 & 16 & 33 \\
\hline 2017 & - & - & 14 & 43 \\
\hline \multirow[t]{2}{*}{2018} & - & - & 9 & 30 \\
\hline & \multicolumn{2}{|r|}{57} & 202 & 215 \\
\hline
\end{tabular}

Anexo 2. Explicación y ejemplo índice de ponderación actividad académica para conformación de muestra fase cualitativa

Para la elaboración de este índice se pidió a un panel de expertos/as -conformado por 6 investigadores y 6 investigadoras seleccionados al azar entre los autores/as incluidos en el universo- que entregaran una ponderación a los siguientes espacios de producción/difusión científica: proyectos con financiamiento público, artículos publicados en revistas nacionales, artículos publicados en revistas internacionales, y ponencias/comunicaciones presentadas en congresos nacionales de la especialidad.

Al panel de experto/as se solicitó responder un cuestionario online con la consigna:

"De los siguientes espacios vinculados a la generación y difusión de conocimiento científico, ¿cómo organizaría o ponderaría su relevancia en el quehacer académico de un investigador o investigadora del campo de la Comunicación en Chile?

- Participación como investigador principal en proyectos con financiamiento público

- Participación como co-investigador en proyectos con financiamiento público
- Participación como autor o co-autor en artículos publicados en revistas internacionales

- Participación como autor o co-autor en artículos publicados en revistas nacionales

- Participación como autor o co-autor en ponencias/ comunicación en congresos

Por favor, asigne a cada ítem un valor, considerando que el total debe sumar $100 \% "$

A través de un promedio de los resultados asignados a cada espacio de producción/difusión científica, se asignó un valor de ponderación:

Con estos valores, se reordenó el universo de investigadores/as aplicando el valor ponderado a cada una de sus participaciones en los espacios de producción/difusión científica identificados en el corpus 1 del proyecto (corpus de investigaciones) 
Anexo 3. Cuestionario entrevista fase cualitativa

\section{Estimado/a investigador/a}

Muchas gracias por aceptar responder esta entrevista que es parte del proyecto "Investigación en Comunicación en Chile: estado del arte y análisis de las características y proyecciones de la comunidad científica nacional" [COD Fondecyt 11160842].

El objetivo del proyecto es caracterizar la comunidad académica que actualmente investiga y genera conocimiento científico sobre Comunicación en Chile. Por lo tanto las preguntas de esta entrevista se referirán tanto a su valoración del campo de la Comunicación en Chile en general, como a su propio quehacer científico en particular.

I.- Validación de datos curriculares [Estos datos solo se validarán, pues la información ya se ha obtenido a través de los sitios institucionales de sus universidades/centros de estudio]

1.- ¿Por qué decidió cursar esos programas de postgrado?

2.- ¿Los realizó con algún tipo de beca para su financiamiento?

3.- La elección del país/institución/o disciplina ¿en qué medida ha aportado a su quehacer como investigador/a?

4.- ¿Cuántos años aproximadamente lleva dedicado/a al trabajo académico y de investigación?

5.- ¿Cómo se generó su decisión de comenzar a trabajar en la academia en general, y en la investigación en Comunicación en particular?

6.- ¿Cómo valora las condiciones actuales en que se desarrolla la investigación en Comunicación en Chile? (financiamiento, jerarquías académicas, credibilidad, autonomía, familia).

II.- Sobre ámbitos, temáticas y enfoques de investigación

7.- ¿Por qué decidió trabajar estos ámbitos, temáticas y objetos/sujetos de estudio? ¿Qué le pareció interesante?

8.- ¿Qué enfoque metodológico y técnicas de investigación ha priorizado en su trabajo? ¿Por qué?

9.- ¿Qué enfoques teóricos utiliza de manera predominante en su trabajo de investigación? ¿Dónde los enmarca disciplinaria y geográficamente?

10.- A lo largo de su carrera, ¿cómo observa que ha ido evolucionando su trabajo como investigador/a? ¿Han variado tus temas de interés, por ejemplo? ¿Por qué?

\section{III.- Sobre las redes de colaboración científica}

11.- ¿Realiza su trabajo de investigación con otros/as académicos/as?

12.- ¿Cómo se ha generado ese trabajo conjunto? ¿Cómo se ha vinculado con estos investigadores/as?

IV.- Sobre el financiamiento y difusión del conocimiento

13.- ¿Cómo ha financiado sus investigaciones? (fondos propios, fondos concursables)

14.- En general, ¿dónde difunde su trabajo de investigación? (Congresos, revistas, otros espacios)
15.- ¿Por qué elige esos espacios?

16.- ¿Cree que sus investigaciones tienen o deben tener algún impacto social más allá de la academia? ¿Cuál?

17.- ¿Cómo se expresa o concreta ese impacto social?

18.- En general, ¿cómo valora la devolución o impacto social que realiza la investigación en nuestro país a las comunidades o grupos que investiga?

V.- Sobre la valoración del campo de la investigación en Comunicación en Chile

19.- En su opinión ¿cómo valora el actual estado de la investigación de la Comunicación en Chile? [En términos epistemológicos e institucionales]

20.- ¿Qué problemas, desafíos y proyecciones visualiza para la investigación en Comunicación en Chile? [En términos epistemológicos e institucionales]

21.- ¿Cómo entiende u observa la relación del campo de la Comunicación con otras área disciplinarias? ¿Hay diálogo conceptual e institucional?

22.- De acuerdo a su experiencia ¿cuáles son las principales tendencias en la investigación en Comunicación en Chile hoy? ¿Cree que hay temáticas "sobre-representadas" y otras con menor presencia de la que "debieran" tener?

23.- ¿Cómo observa el lugar de su propio tema de interés en el campo de la investigación en Comunicación en Chile?

VI.- Sobre la perspectiva de género (en los investigadores/as y en la investigación)

Como parte de este estudio hemos querido incorporar la variable género para comprender el campo de la investigación en Comunicación en Chile.

24.- ¿Considera que existen diferencias de género en la producción científica/académica en el área de la comunicación en Chile?

25.- Hace un momento le preguntamos por las condiciones actuales en que se desarrolla la investigación en Comunicación en Chile ¿Observa diferencia en estas condiciones entre investigadores e investigadoras?

26.- Si observa alguna diferencia, en su opinión ¿qué se podría hacer para superar con estas diferencias de género?

27.- En sus investigaciones ¿considera las variables de género como un aspecto relevante? ¿Por qué?i

VII.- Sobre los referentes del campo de la Comunicación en Chile

28.- ¿Qué investigador/a destacaría por su trabajo en el campo de la investigación en Comunicación en Chile hoy? ¿Por qué?

29.- ¿Qué académico destacaría por su aporte "histórico" (de investigación o teórico) al campo de la investigación en Comunicación en Chile? ¿Por qué?

Muchas gracias 